\title{
The virtual cathode as a transient double sheath
}

T. Intrator, M. H. Cho, E. Y. Wang, N. Hershkowitz, D. Diebold, and d. Dekock

Department of Nuclear Engineering and Engineering Physics, University of $\mathrm{Wisconsin-Madison}$, Madison, Wisconsin 53706

(Received 29 October 1987; accepted for publication 6 June 1988)

The two-dimensional plasma potential measurements are given of a space-charge dominated double sheath near a hot cathode. Laboratory data show that a virtual cathode is a selfconsistent solution only for transient cathode plasma system. Slow charge exchange ions get trapped in the potential dip that forms the virtual cathode and eventually destroy it.

\section{INTROOUCTION}

Even though an electron emitting cathode may ideally be capable of emitting large electron currents, the electric field generated by the associated "space charge" self-limits the maximum possible electron emission from the cathode. The practical operation of cathode related devices aepends on how the space charge and electric fields interact. Whise there have been many investigations ${ }^{1}$ of diode circuit characteristics and theory, ${ }^{2-4}$ there have been very few experimental measurements ${ }^{2,5,6}$ of the electric fields inside this spacecharge dominated volume. In this paper we present laboratory measurements of the local electric field in a spacecharge dominated region.

The virtual cathode is a region of zero electric field where an excess of local electron charge has accumulated. This is shown schematically for a plasma-filled half-piane in Fig. 1(a). The eiectric field vanishes where the local plasma potential, $\phi_{p}(z)$, has a minimum, $\phi_{m}$. Poisson's equation requires that excess electron space charge occupy the region where $d^{2} \phi_{p} / d z^{2}>0$, i.e., for $0<z<z_{\text {inf }}$. The net charge is zero at the inflection point. In the cathode region, the electric field that retards the electron source emission joins the (oppositely directed) electric field that extracts elections. There is a net electron charge surplus in the virtual cathode region which adjoins a region of ion charge surplus in a separate sheath that attaches the virtual cathode to the quasineutral plasma. These two non-neutrai regions form the two portions of a double sheath.

The virtual cathode impedes current fiow from both the cathode and background plasma. In Fig. I(b) the balance between the plasma charged particle currents is shown schematically. Cathode current is divided between trapped $\left(I_{c, t r}\right)$, and escaping $\left(I_{c, e s e}\right)$ portions. Plasma thermal electron current is partialy attenuated and transmitted $\left(I_{e, \mathrm{th}}\right)$, and plasma ion current $\left(L_{i}\right)$ travels through the virtual cathode region.

In this paper, we present laboratory measurements of the plasma potential near a hot, electron emitting cathode. When space charge begins to affect the cathode emission, we demonstrate that the plasma potential finds a transient (60IE) solution that corresponds to the Langmuir spacecharge double sheath. ${ }^{3}$ We also demonstrate that the transient solution is much different from the steady-state one. In the presence of an ion beam, the sheath region extends very far (30-50 Debye lengths) from the cathode.
Potential dips that separate two plasma regions with different plasma potentials can be found in many situations, both in nature and in the laboratory. These include moving "ion acoustic" double layers in space" and in the laboratory," positively biased probes in plasmas, and stationary thermal barriers in tandem mirrors. "The stationary structures share the characteristic that their potential wells eventualy must fill up with ions that either scatter into the region or are born there by charge exchange. Potential dips that are isolated in three dimensions should not persist in steady state.

On the other hand, stationary (in time and space) potential wells have been achieved near positively biased plasma boundaries ${ }^{10}$ by the addition of ion "pumping" which removes trapped ions. For example, this can occur along the third dimension of a two-dimensional potential dip.

We describe the experiment in Sec. II, and the diagnostic techniques in Sec. II. Addition of a negative current bias to an emissive probe is shown to be useful for measurements of $\phi_{p}$ in a sheath as well as a quasineutral plasma. In Sec. IV we show mesurements of $\phi_{p}$ in parallel and perpendicular (to the planar cathode) directions for different cathode emission and plasma conditions. In Sec. V the familiar steady-state Child-Langmuir solution is shown to not be self-consistent in a plasma which contains significant neutral densities because the potential dip flls up with charge ex-

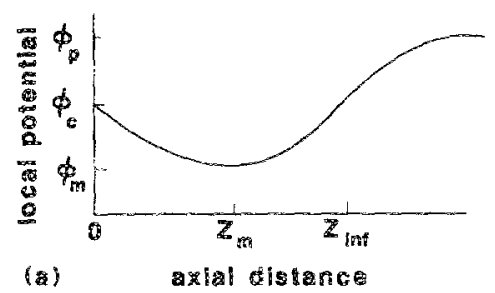

FIG. 1.(a) Schematic potential shape $\phi(z)$ in cathode space-charge region showing cathode $\phi_{c}(z=0)$, ninimum $\phi_{m}\left(z=z_{m}\right)$, and infection point $\left(z={ }_{\mathrm{inf}}\right)$ plasma potentials $\left(\phi_{n}\right)$. (b) Schematic drawing of the zero net current balance for a floating surface, such as an emissive probe or hot cathode, showing escaping $\left(I_{c, \text { es: }}\right)$ and trapped $\left(I_{r, t z}\right)$ cathode electron current balanced by collected plasma electron thermal cirrert much larger than ion current $\left(I_{e, t \mathrm{t}} \geqslant I_{i}\right)$. 


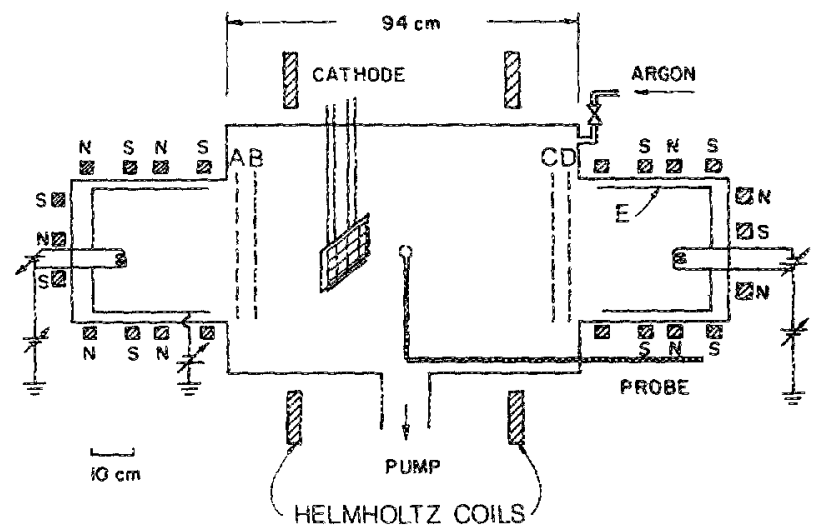

SOURCE

TARGET

SOURCE 2

FIG. 2. DOLI II, the experimental triple plasma device. The hot cathode grid and two-dimensionally scannable emissive probe are shown schematically.

change ions and disappears. In Sec. VI we show that ions can also be pumped out to nearby boundaries, and close with a discussion in Sec. VII.

\section{EXPERIMENT}

This experiment takes place in the target chamber of a triple plasma device, DOLI II (Ref. 11), shown in Fig. 2 . The plasma is created by an argon muitidipole filament discharge in one of the source chambers. The flow of this plasma into the target chamber is controlled by biased grids $A-D$ (each approximately $60 \%$ transparent to plasma, $90 \%$ optical transparency). The target density is controlled by source density, and target potential is determined by eiectron and ion flow into the target from the right-hand chamber and the boundary grids.

The target plasma is usually operated in the collisionless regime, except for the data at highest neutral pressure $\left(\lambda_{\mathrm{en}}\right.$ $\sim L_{D}, P_{0} \sim 10^{-3}$ Torr). The thermal electron-neutral collision mean free path $\left(\lambda_{\mathrm{er}}-600 \mathrm{~cm}, P_{0}=1 \times 10^{-4}\right.$ Torr argon, $1-3 \mathrm{eV}$ electrons) is much larger than the device dimensions $\left(L_{D}=60-90 \mathrm{~cm}\right)$. The electron temperature $\left(T_{e} \sim 1-\right.$ $1.5 \mathrm{eV})$ is much higher than the ion temperature $\left(T_{i} \approx 0.3\right.$ eV). Device parameters are summarized in Table $I$.

A hot cathode is operated in the center of the target chamber. It consists of a $4 \mathrm{~cm} \times 4 \mathrm{~cm}$ square of thoriated (2\%) tungsten mesh ( $90 \%$ opticaily transparent, $60 \%$ plasma transparency) with wires $(0.0025 \mathrm{~cm}$ diam) spaced on 0.025 -cm centers. The tantalum electrical contacts and support structure have a collecting area of $6 \mathrm{~cm}^{2}$ that collects, but does not thermionically emit electrons. This cathode can be heated white hot to electron themionic emission with half-wave rectified $60-\mathrm{Hz}$ ac power. Most data are taken during the off cycle, when there is zero voltage across the cathode.

The whole cathode can either be biased through a low impedance with respect to ground $(<1 \Omega)$ and allowed to draw current, or floated through a high impedance with re-
TABLE I. Parameters of the experimental device DOLI II and characteristics of the plasma in the target region.

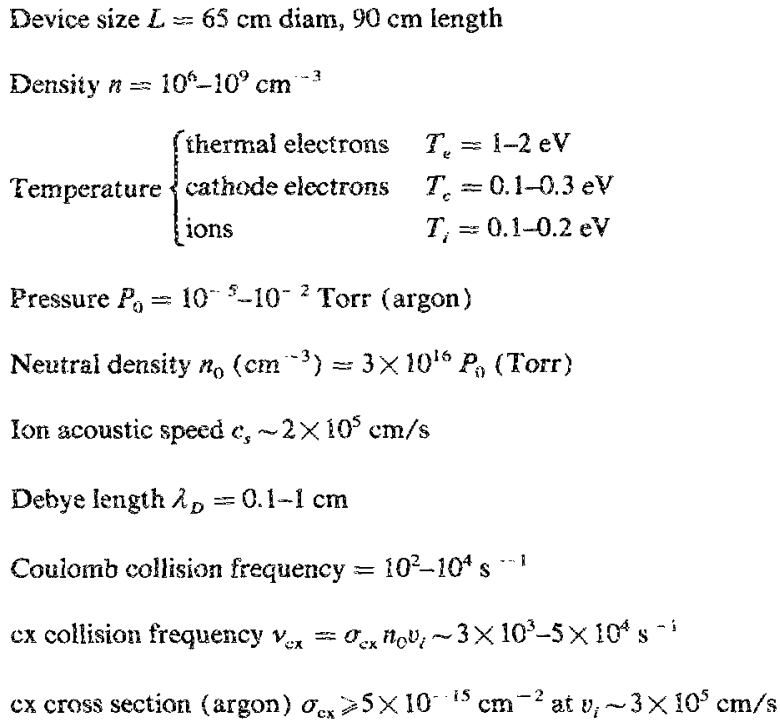

spect to ground ( $10^{11} \Omega$ maximum for the operational amplifier) so that no current flows. The data shown in this paper show the local plasma potential for the foating condition. This zero total current is a balance between collected plasma current and emitted cathode current, shown schematically in Fig. 1 (b). Electrons get emitted from the hot cathode; some escape the potential barrier shown in Fig. 1(a), and some reflect and are reabsorbed by the cathode. The reflected cathode electrons form a trapped population which depresses the local plasma potential. The ion current is usually much smaller than the electron currents originating either at the cathode or plasma. The plasma electron fux is also attenuated by the potential barrier. The flux balance is dominated by the attenuated currents from both cathode and plasma.

The boundary grids can be biased to allow the entry of drifting populations of electrons or ions. The ion beams that are injected into the target chamber have a smaller density than the thermalized ion background, as shown below.

The ion beam density can be estimated if fux is conserved by the accelerated beam between the source chamber and target chamber, and the ion and electron charge density approximately cancel in the quasineutral portions of the source and target. Then for the ion beam, $n_{b} v_{b}$ (target) $=\frac{1}{2} \times n_{\text {src }} c_{s}$ (source), where $n_{b}$ and $v_{b}$ are, respectively, the target beam density and speed. The source density $\left(n_{\mathrm{src}}\right)$ was measured from Langmuir probe measurements of electron saturation current to be approximately to be 3 times the target density $\left(n_{\mathrm{tgt}}\right)$. The source speed is $c_{s}=\left(T_{e} / m_{i}\right)^{1 / 2}$ and the ion beam energy is approximately $E_{b} \simeq 3 \mathrm{eV} \simeq 2 T_{e}$, as measured by the plasma potential drop between the quasineutral regions of source and target plasmas. These parameters are characteristic of the data discussed in Sec. $Y$.

In the target, the ratio of the ion beam $\left(n_{b}\right)$ to total electron density $\left(n_{\text {tgt }}\right)$ is approximately

$$
n_{b} / n_{\mathrm{tgt}} \sim\left(c_{s} / 2 v_{b}\right) T^{2}\left(n_{\mathrm{src}} / n_{\mathrm{tgt}}\right) \sim 0.24
$$


where the flux conserving beam density gains energy $E_{b}$ and is attenuated during acceleration to $n_{b} / n_{\mathrm{scc}}=\frac{1}{2}\left(1+2 E_{b} /\right.$ $\left.T_{e}\right)^{-1 / 2}$. The grid transparency is approximately $T \sim 0.6$.

\section{DIAGNOSTIC TECHNOUES}

In this experiment, we employ emissive probes to measure the local plasma potential. Emissive probes can be used to measure the plasma potential in a variety of plasmas, over a wide range of densities and electron temperatures. ${ }^{12-15} \mathrm{Un}$ like collecting probes, emissive probes are not sensitive to any plasma drifts. In this experiment, the range of drift energies is comparable to the magnitude of the potential variations.

Therefore, emissive probes were used to provide unambiguous potential data. The emissive probes had thoriated Tungsten filaments of $2.54 \times 10^{-3} \mathrm{~cm}$ diam with lengths ranging from 0.25 to $1.0 \mathrm{~cm}$. The spatial resolution is limited axially by the filament length, and laterally by the filament support structure width, to no better than $0.2 \times 0.2 \mathrm{~cm}^{2}$. The probe structure was electrostatically shieided up to the tip, and there was a ceramic coating on the nonemitting portions of the wires. Grounded surfaces on the probe assembly were kept far away from the measurement region.

The most reliable method of determining the plasma potential involves the inflection point of the current-voltage trace of a hot electron emitting filament, in the limit of zero emission. ${ }^{12}$ The probe flament is heated with a $60-\mathrm{Kz}$ halfwave rectified heating voltage. We typically sweep the probe voltage bias in $100 \mu$ during the of cycle of the probe and hot cathode heaters, while the probe is still hot and emitting, but has no voltage across it.

In the limit of zero electron emission, this technique is accurate to a fraction of an $\mathrm{eV}$ (hot wire temperature), and does not have the space-charge problems associated with strong electron emission. Since the potential diagnostic is used in the limit where space-charge effects vanish, we can measure potentials in the non-neutral cathode region. The space-charge effects observed in this experiment are due to the hot cathode plasma system, and not to inadequacies of the diagnostic technique.

One other technique was used, when time-resolved potential data were required. The probe was connected to the noninverting input of a unity gain, high impedance voltage follower as shown in Fig. 3 (Tektronix Type 0 op amp-piug in) with the switch open. Since the input impedance of the

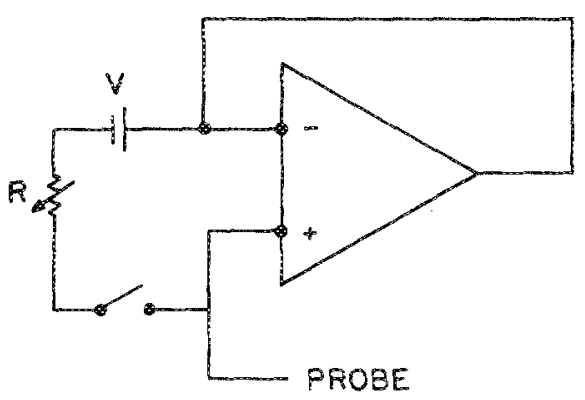

FIG. 3. High inpedance voltage follower (with switch open) with adjustable low impedance currert source (switch closed). op-amp was much larger than the sheath impedance of the probe $\left(Z_{\text {sheath }} \leqslant 1 M \Omega\right)$, the probe draws virtually no current.

Negative current was added to the probe ${ }^{15}$ (Fig. 3 with switch closed) to move the probe floating potential (voltage at which the probe draws zero current) to a lower impedance operating point on the I-V curve. In Fig. 4 the triangles show the local potential near the hot cathode as measured with the infection point-zero emission technique. The cathode is located at $z=0 \mathrm{~cm}$. These data are used to calibrate the appropriate negative current bias by comparison of foating point data at different bias currents (lines) with reference measurements(triangles). It is apparent that the correct value of negative bias current moves the floating potential very close to the plasma potential. The error appears to be only a fraction of a volt.

Negative current bias also improves the time response. In a low density plasma such as in this experiment, little plasma electron current is available to charge the probe negative as it follows a negative going plasma potential. ${ }^{16} \mathrm{Addi}$ tional bias current reduces the charging time for the probe voltage. The parallel capacitance of the sheath and detector circuit is charged by the sum of plasma and bias currents. The plasma current can be attenuated by space-charge effects, as will be shown later in Sec. IV. Figure 4 illustrates the usefulness of the current bias method in a non-neutral sheath region, as well as quasineutral plasma regions.

\section{THE POSSIBLE POTENTIAL SHABES}

Equipotential contours near the cathode are shown in Fig. 5. Even though the experimental geometry is one di-

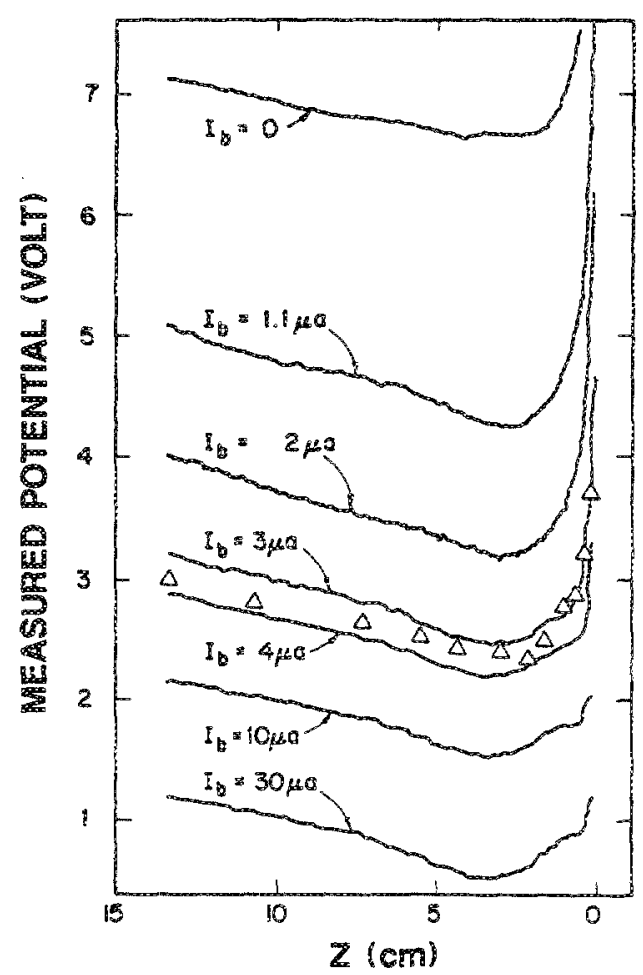

FIG. 4. A comparison of the negative current biased foating probe (lines) with the inflection point technique (triangles). An absolute calibration provided by inffection point data shows that absolute (relative) plasma potential changes can be deduced with (without) the correst offset current. 


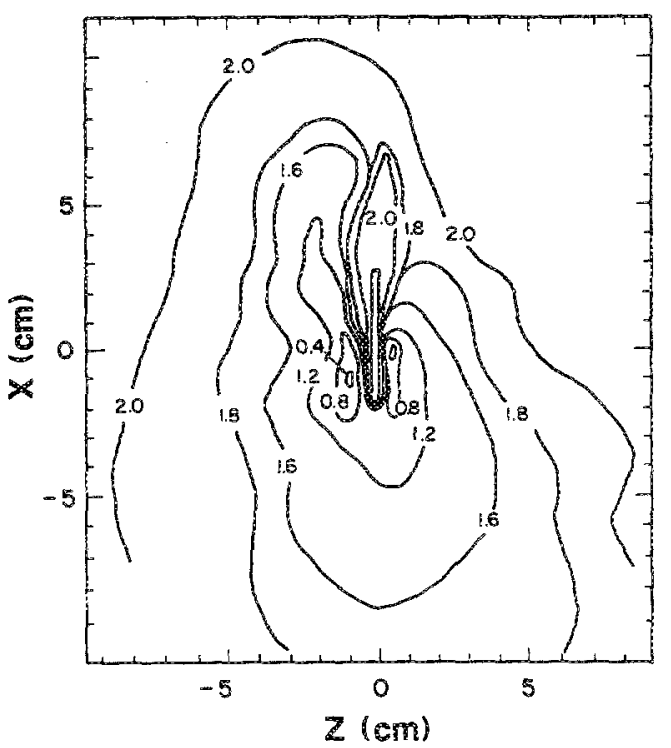

FIG. 5. Equipotential contours shown in a two-dimensional plot of the region local to the planar hot cathode, where $\lambda_{B} \sim 0.7 \mathrm{~cm}$. This planar boundary immersed in a homogeneous plasma does not have a planar sheath.

mensional (Debye length $\lambda_{D} \ll$ cathode size $L_{c}$ ), the potential features extend at least a distance of $L_{c}$ in two dimensions. The planar cathode is an equipotential surface, floating at $V_{c}=+2.3 \mathrm{~V}$. Equipotential contours are parallel near the cathode, and decrease to the local potential depression that is also parallel to the cathode. For these data the Debye length $\lambda_{D}$ varies from approximately $0.1 \mathrm{~cm}$ for the cathode electrons to $0.9 \mathrm{~cm}$ for the plasma thermal electrons. A large potential dip is localized between 0.5 and 1.0 $\mathrm{cm}$, or several $\lambda_{n}$ from each side of the cathode surface. All of the equipotential contours show a two-dimensional depression in the local plasma potential that completely surrounds the hot cathode. Note that the contours are closed, so that every electron that originates at the cathode must see some confining potential, for all trajectories.

The data in Fig. 5 indicate large spatial density variations for cathode electrons and small spatial inhomogeneities for plasma thermal electrons, assuming the Boltzmann relation holds for these populations. The variations of $e \phi_{p}$ are larger than the thermal energy $\left(T_{\mathrm{ec}}\right)$ of the Maxwellian cathode electrons, and smaller than the background electron thermal energy $\left(T_{e}\right)$.

The hot cathode is an equipotential surface, floating more positive than the background plasma, but the magnitude of the potential dip is not constant around the cathode. The up-down asymmetry seen in Fig. 5 may be a result of nonuniform emission from the mesh, or a cathode surface that was not perfectly flat. Variations in local surface temperature of the tungsten mesh could result in different thermionic emission along the grid. The Richardson-Dushmann ${ }^{17}$ formula is

$$
J_{c}\left(\mathrm{~A} / \mathrm{cm}^{2}\right)=A T_{c}^{2} \exp \left(-\phi_{w} / T_{c}\right),
$$

and can be evaluated for tungsten-doped thorium, where $A=3.0 \mathrm{~A} \mathrm{~cm}^{-2} \mathrm{~K}^{-2}, \phi_{w}=2.63 \mathrm{eV}$ is the work function,

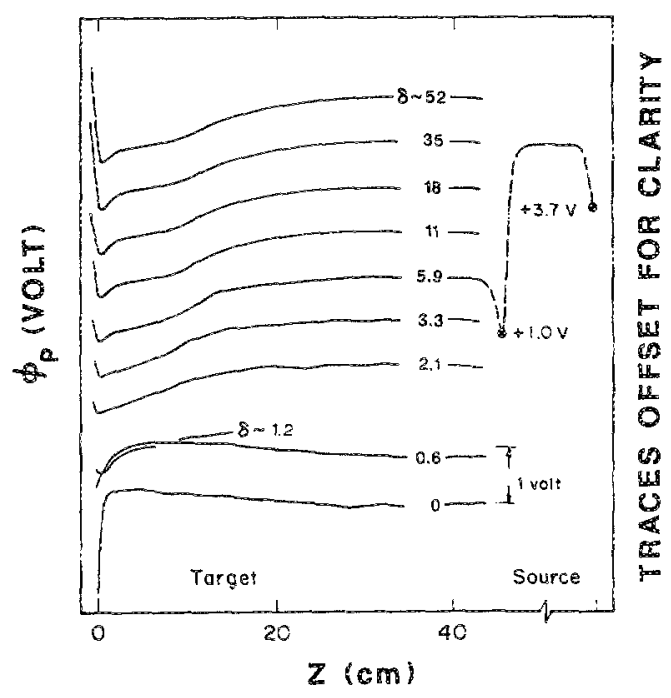

FIG.6. Axial scan of the plasma potential on a $z$ axis eut through a structure like that shown in Fig. 5. The cathode emission parameter is $\delta$.

and $T_{c} \sim 1300 \mathrm{~K}$ is the cathode temperature. This predicts a $50 \%$ change in emission for a $2 \%$ change in $T_{c}$. The asymmetry may also be due to potential variation in the third $(z)$ dimension (out of the $x-y$ plane in Fig. 5), as alluded to in Sec. VI.

An axial cut across this data set at $x=0$ gives the plasma potential profiles that are considered in the balance of this paper. Examples of the one-dimensional potential profles along the axis of the cathode are shown in Fig. 6, which gives plots of $\phi_{p}(z)$ vs $z$. Each successive curve is for increasing values of cathode temperature, which correspond to increasing vaiues of the emission parameter $(\delta)$. The curve labeled $\delta \sim 5.9$ has the boundary conditions explicitly shown and is for plasma conditions similar to the Fig. 5 plasma.

The source plasma on the right is injecting an ion beam with energy of approximately $3 \mathrm{eV}$. The target grids (grids B and $C$ from Fig. 2) were set to $+1.0 \mathrm{~V}$, and the source grid and anode (grid D and anode $E$ in Fig. 2) were held at +3.7 $\mathrm{V}$, respectively. The quasineutral plasmas float positive of their respective boundaries. For Fig. 6 , every trace has the same parameters, but only the $\delta \sim 5.9$ trace is drawn in its entirety. The right-hand side of every curve in Fig. 6 corresponds to the same potential, but each curve is offset vertically by $1 \mathrm{~V}$, for clarity of presentation.

The emission parameter $(\delta)$ is defined as the ratio of the maximum cathode current that can be emitted into the plasma divided by the thermal electron collection current to the cathode. We can calculate $\delta$ from a current-voltage characteristic for the cold and hot cathode as shown in Fig. 7. The cathode bias voltage ( $\left.V_{\text {bias }}\right)$ was swept with respect to the experiment ground. For large negative bias with respect to the plasma potential far from the cathode [ $\left(e \phi_{p}^{(\infty)}-e V_{\text {bis }}\right)$ $\geqslant T_{e c}$ but less than an ionization energy] the sheath potential decreases from plasma to cathode similar to that illustrated in Fig. 6 for $\delta=0$. Since all of the cathode electron current escapes to the plasma, one can measure the maximum hot cathode emission. 


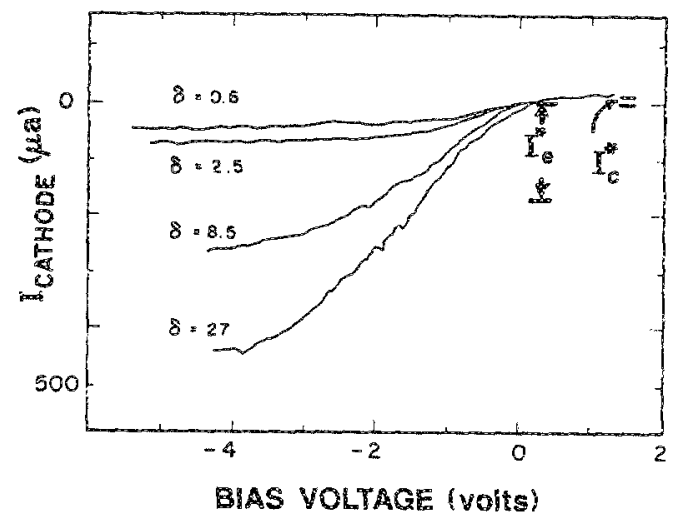

FIG. 7. Current-voltage characteristic of the hot cathode electron emitter for several values of $\delta$. Maximum collected plasma current is shown by $I_{c}^{*}$, ind for $\delta=8.5$, emitted current $I_{e}^{*}$ is shown.

Returning to Fig. 6 , it is apparent that the plasma potential near a floating cold boundary $(\delta=0)$ is much different from the potential near the electron emitting boundary $(\delta=52)$. For $\delta=0$ the sheath extends for several Debye lengths. The space-charge region for $\delta$ approaching unity already exhibits the double sheath structure, and for $\delta>2$, becomes quite large $\left(30-50 \lambda_{D}\right)$ and includes a double layer that joins to the bulk plasma. The virtual cathode where $E=-\nabla \phi_{p} \sim 0$, is a potential barrier that attenuates electron currents from both the plasma side and the cathode side. This virtual cathode can be seen near the cathode for $8>1.2$. For high enough emission, a double layer occurs ${ }^{18}$ when a streaming ion population is present far from the cathode. For the data shown in Fig. 6 a beam with energy of approximately $3 \mathrm{eV}$ and density approximately $2 \times 10^{5} \mathrm{~cm}^{-3}$ was present.

The cathode-virtuai cathode structure is consistent with the Child-Langmuir limit and the total current balance of the floating cathode. This is shown in the Appendix, where the Boltzmam attenuation of cathode emission is calculated in terms of the potential barrier between cathode and virtual cathode.

\section{WHAT IS A SELF-CONSISTENT STEADY STATE?}

Given this one-dimensional picture, we should wonder why the potential dip does not fill up with slow ions. Charge exchange, for instance, preferentially produces slow ions at the expense of fast ions because the scattering rate $v_{c x}$ $=n_{0}\left\langle\sigma_{\mathrm{cx}} v_{\mathrm{inn}}\right\rangle$ scales with ion velocity ${ }^{19}$ and all resulting ions have the (low) energies of the neutral population. The competing collision process, ion Coulomb scattering, is much less significant for these experimental parameters $\left(E_{b}\right.$ $\sim 3 \mathrm{eV}, n_{0} \sim 2.25 \times 10^{12} \mathrm{~cm}^{-3}$, argon gas ${ }^{18} \sigma_{\mathrm{cx}} \sim 5 \times 10^{-1.5}$ $\left.\mathrm{cm}^{2}\right)$. The charge exchange time $\left(v_{\mathrm{cox}}^{-1} \simeq 300 \mu \mathrm{s}\right)$ is much less than the Coulomb scattering time $\left[\left(\omega_{p i}^{2} e^{2} / v_{i}^{3} m_{i}\right)^{-1} \simeq 1 \mathrm{~s}\right]$.

We apply a large current $(40 \mathrm{~A})$ and voltage $(20 \mathrm{~V})$ to heat the hot cathode. During the heating cycle the ions get dumped out of the dip to the plasma when the one side of the cathode is much more positive than the nearby piasma po-

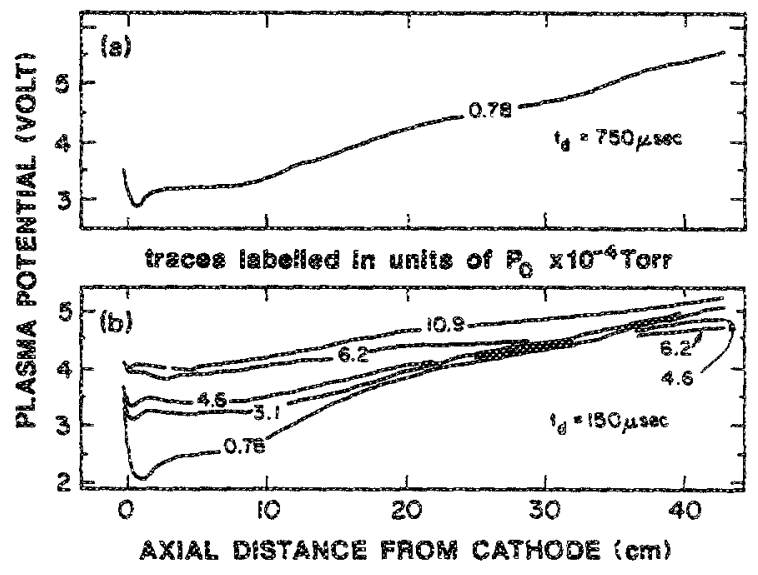

FIG.8.(a) Axial scans of plasma potential $\phi_{\rho}$ (z) vs $z$ for increasing neutral pressure, labeled in units of $10^{-4}$ Torr. Time delay was $150 \mu \mathrm{s}$. (b) Similar axial scan of $\phi_{p}$, but for longer time delay $t_{t}=750 \mu \mathrm{s}$.

tential. Ions also fall simuitaneously into the negative side of the cathode. The presence of this particle pumping mechanism maintains the potential dip. The experimental region is purged of particles periodically at $60 \mathrm{~Hz}$, and then a time delay of at least $150 \mu \mathrm{s}$ is allowed for the sheath system to relax. Emissive probe data can then be taken using either boxcar sampling techniques for the current bias method or the inflection point method. The region which initially was an ideal electron-rich virtual cathode subsequently evolves towards a quasineutral plasma as it fills with slow lons.

In Fig. 8(a) several plasma potential profiles are shown for increasing neutral pressure. The charge exchange rate, which is proportional to $P_{0}$, increases by more than an order of magnitude as the neutral pressure is increased. The resulting charge exchange ions destroy the potential dip. This is not surprising because even for moderate neutral density $\left(P_{0} \sim 3 \times 10^{-4}\right.$ Torr $)$ the mean free path for a charge exchange event $\left(\lambda_{c \kappa} \leqslant 20 \mathrm{~cm}\right)$ is approximately the size of the potential structure. In practice, it is very difficult to design a laboratory experiment that is free from charge exchange effects. For our data the charge exchange time varies from 300 $\mu \mathrm{s}\left(P_{0}=7.5 \times 10^{-5}\right.$ Torr $)$ to about $20 \mu \mathrm{s}\left(P_{0}=1.1 \times 10^{-3}\right.$ Torr).

The data in Fig. $8(a)$ were taken at $150 \mu$ s after the cathode heater turns off. An axial profile of plasma potential $\phi_{p}(z)$ for a longer delay time of $t_{d}=750 \mu \mathrm{s} \sim 2.3 \tau_{\mathrm{ex}}$ is shown in Fig. 8(b). This delay time corresponds to similar traces in Fig. $8(a)$ where the normalized delay time is aiso approximately the same. For example $t_{d} / \tau_{\mathrm{cx}} \sim 150 \mu \mathrm{s} / 73$ $\mu s \sim 2$ for the trace at $P_{0}=3.1 \times 10^{-4}$ Torr. The de level and the net curvature of the potential profile are similar to that of Fig. $8(\mathrm{~b})$. The transient lifetime of this nonmonotonic potential apparently depends upon a particle scattering time.

In this experiment, we can see that charge exchange ions eventually dominate the behavior of the virtual cathode potential dip. The idealized steady-state model of nonmonotonic potentials is only valid while the effects of particle sources and sinks are not significant. 


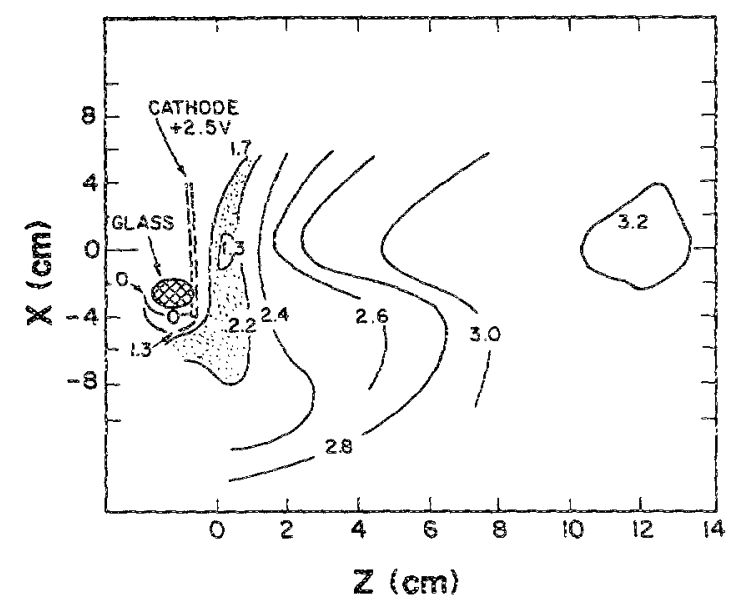

FIG.9. Two-dimensional graph of equipotential contours near an electron emitting cathode when an ion pumping boundary, i.e., a glass insulator support, is nearby. Note that the shaded potential dip is much more exaggerated than the similar diagram shown in Fig. 5 , in which ion pumping boundary was moved far away.

\section{TWO-DIMENSIONAL CONTOURS}

It was found that the potential dip near the cathode can either be small and localized to the cathode, as in Fig. 5, or have a large transverse size and connect to the floating glass insulators that cover the cathode support, as in Fig. 9. The shaded area in Fig. 9 indicates the potential dip which is connected by a monotonic decreasing potential to the glass boundary. Ions can thus be iost (pumped out) along equipotential contours that terminate on the glass. In Fig. 9 the glass insulator was very close to the cathode; in Fig. 5 the insulator was moved far way. A comparison of Figs. 5 and 9 , where a much larger solid angle was subtended by the glass insulator in Fig. 9, shows that the main difference is that the large magnitude dip is less localized in Fig. 9.

The up-down asymmetry that dominates the data in Fig. 9 is reminiscent of that seen in Fig. 5. This suggests that three-dimensional current paths, even to the distant foating boundaries in Fig. 5, can still affect the potential local to the cathode.

\section{CONCLUSION}

It is well known that space-charge effects exist near strongly emitting electron sources. Onily recently has it been recognized that self-consistent electric fields depend on particle sources and particle pumping in ways that are far from ideal. In this laboratory experiment, we show that the virtual cathode as introduced by Langmuir is self-consistent only for a transient cathode-plasma system if there is significant neutral density. Charge exchange ions fall into the potential dip that forms the virtual cathode and eventually destroy it. We believe that the data shown in this paper comprises the first two-dimensional measurements of the electric field inside a cathode-space-charge-limited system.

Our laboratory model of the electron emitter is a planar hot cathode that can emit thermionically far in excess of the
Child-Langmuir limit if biased appropriately. Since the cathode was foating, a zero current balance between virtual cathode electron emission and collected plasma current was maintained. We have measured the local plasma potential using electrostatic emissive probes in the space-charge region for a large range of emission and background plasma concitions. When the neutral pressure $\sim 10^{-3}$ Torr, spacecharge effects dominate a large $\left(L>30-50 \lambda_{D}\right)$ two-dimensional region near the cathode that extends for many Debye lengths.

Even though zero current is flowing in this system, there are significant transient electric fields inside the diode spacecharge region. The double sheath that connects the non-neutral virtual cathode and the quasineutral plasma is shown in detail. In the presence of an ion beam an extra double iayer appears between the double sheath and the quasineutral plasma.

The spatial extent of space-charge effects and the size of the self-consistent potentials involved are usually difficult to calculate realistically. For this reason, space-charge effects are frequently ignored when the plasma-wall-sheath problem is considered. In this experiment, potential dips much larger than the cathode temperature are observed for a wide range of cathode emission and background plasma and beam conditions. Secondary electron emission can aiso generate similar effects to the ones shown in this experiment. ${ }^{20}$

Measurements of the plasma potential were obtained with enissive probes. Aside from the reference inflection point technique, we also could add negative current bias so that an emissive probe floats at a potential very close to the plasma potential. Although we did not originate this current bias technique, ${ }^{15}$ we do show that it is reliable both in the quasineutral plasma and non-neutral sheath.

\section{ACKNOWLEDGENTS}

This work was supported by NSF Grant No. ATM8611161 and NASA Grant No. NAGW-275.

\section{APPENDIX}

The cathode-virtual cathode structure is consistent with the Child-Langmuir limit and the total current balance of the floating cathode. To show this, we will first look at the plasma side of the virtual cathode by calculating the spacecharge-limited emission allowed by the virtual cathode in

TABLE IF. Tabulation of the ratio of Child-Langmuir emitted current to collected background electron current for the potential structures shown in Fig. 6 .

\begin{tabular}{cc}
\hline$\delta$ & \multicolumn{1}{c}{0.36} \\
3.3 & 0.74 \\
5.9 & 1.25 \\
11.0 & 0.9 \\
18.0 & 1.1 \\
35.0 & 0.62 \\
52.0 &
\end{tabular}


TABLEMI. A comparison of ideal cathode emission $(\delta)$ and attenuation of cathode current by the virtual cathode potential dip.

\begin{tabular}{ccc}
\hline$\delta$ & $\exp \left(e \phi_{c \mathrm{~m}} / T_{c}\right)$ & $\delta \exp \left(-e \phi_{\mathrm{cm}} / T_{e}\right)$ \\
\hline 1.2 & 0.59 & 0.71 \\
2.1 & 0.35 & 0.74 \\
3.3 & 0.29 & 0.95 \\
5.9 & 0.19 & 1.1 \\
10.4 & 0.094 & 0.93 \\
18.0 & 0.074 & 1.3 \\
\hline
\end{tabular}

Fig. 6. In one dimension, the Child-Langmuir law ${ }^{3}$ states that the maximum current that can be emithed into a vacuum is

$$
I_{\mathrm{CL}}=\frac{4}{3}(2 e / m)^{1 / 2}\left(\phi_{\mathrm{ms}}^{3 / 2} / 4 \pi d^{2}\right) A_{\mathrm{em}},
$$

where $\phi_{\mathrm{mat}}=\left|\phi_{\min }-\phi_{\mathrm{steati}}\right|, \phi_{\text {sheath }}$ is the potential at the inflection point between the virtual cathode and the double layer. $A_{\mathrm{em}}$ is the emitting area of the virtual cathode. For a floating emitter, the emission current balances collected plasma thermal current

$$
I_{e, \mathrm{th}}=n_{\mathrm{e}} v_{e, \mathrm{th}} \exp \left(-e \phi_{m p} / T_{e}\right) A_{\mathrm{c}} / A_{\mathrm{s}}
$$

where $\phi_{m p}$ is the barrier potential $\left|\phi_{\mathrm{min}}-\phi_{p}\right|$, and $A_{c} \sim 11$ $\mathrm{cm}^{2}$ is the collecting area of the cathode grid $\left(16 \mathrm{~cm}^{2}\right.$ grid $\times 40 \%$ piasma opacity $+6 \mathrm{~cm}^{2}$ of support structure), and the electron density is given by $n_{e, t h}$, and electron thermail speed by $v_{e, t h}$. The ratio is

$I_{\mathrm{CI}} / I_{\mathrm{e}, \mathrm{tb}}=(16 \sqrt{2} / 9)\left(\Phi_{\mathrm{ms}}^{3 / 2} / \mathrm{s}^{2}\right) \exp \left(\Phi_{\mathrm{mp}}\right) A_{\mathrm{em}} / A_{\mathrm{c}}$,

where the normalized potential is $\Phi \equiv e \phi / T_{e}$ and $s \equiv d / \lambda_{D}$ is the normalized distance $(3 \mathrm{~cm})$ over which the cathode extraction field is approximately constant, and not the extra double layer. For high enough emission $(\delta>3.3)$, the current that can be emitted from the virtual cathode is approximately the collected plasma current, as tabulated in Table 11 . From the data in Fig. 6 , for example at $n_{\mathrm{c}} \sim 10^{6} \mathrm{~cm}^{-3}, 1$ $\mathrm{cm}<z<3 \mathrm{~cm}$, the Debye length is $\lambda_{2} \sim 0.7 \mathrm{~cm}, T_{e} \sim 1-1.5$ $\mathrm{eV}, s \sim 1, \Phi_{m s} \sim 0.13-0.3$, and $\Phi_{m p} \sim 0.8-1.1$. Electrons can also leak out of the virtual cathode region, in the other two dimensions ignored in this model, so that this version of the Child-Langmuir law does not account for the entire current balance.

The potential barrier between the cathode and virtual cathode is also consistent with the previous determinations of $\delta$ as shown in Fig 7. Since the potential between the cathode and virtual cathode has positive curvature, the potential dip contains excess electron charge density. The escaping current is assumed to attenuate exponentially for Maxwel- lian cathode emitted $\left(I_{s}\right)$ electron current and should be approximateily equal to $I_{\mathrm{CL}}$ and $I_{e, t h}$ so that

$$
I_{c, \mathrm{esc}}=\exp \left(-e \phi_{\mathrm{cm}} / T_{c}\right) I_{c}
$$

where $\phi_{\mathrm{cs}}=\phi_{\mathrm{c}}-\phi_{\mathrm{min}} \geqslant 0$.

For all the traces in Fig. 6 where $\delta=I_{c} / \lambda_{e, t h} \leqslant 11$ the product of independently determined $\delta$ and $\exp \left(-e \phi_{\mathrm{cm}} /\right.$ $\left.T_{c}\right)$ is of order unity $( \pm 30 \%)$. Data from Fig. 6 are used to compare these two quantities in Table $\mathrm{IH}_{y}$ where $T_{c} \sim 0.3$ $\mathrm{eV}$. The potential barrier between cathode and virtual cath. ode is approximately self-consistent with the Child-Langmuir law and total current balance of the floating cathode.

${ }^{1}$ N. St. J. Eraithwaite and J. E. Allen, Int. J. Electron. 51,637 (1981); J. Enriques, G. B. Righetti, F. Magistrelli, and A. Boschi, Nuovo Cimento 38, 26 (1965); M. T.C. Fang, D. A. Frazer, and J. E. Allen, Brit. J. Phys. D 2, 229 (1969).

${ }^{2}$ C. D. Child, Thys Rev. 32, 492 (1911).

${ }^{3}$ I. Langmuir, Phys. Rev. 2, 6, 450 (1913); ibid. 28, 727 (1926); I. Lang-

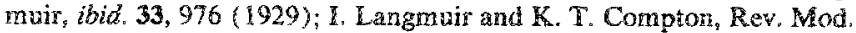
Phys. 3, $191(1931)$.

4. Pierce, J. Appl. Phys. 15, 721 (1944); C. K. Birdsall and W. B. Bridges, Electron Dynamics of Diode Regions (Academic, New York, 1966); J.G. Andrews and J. E. Allen, Proc. Royal Soc. London A 320, 59 (1971); V. L. Sizonenko, Sov. Phys. Teeh. Phys. 26, 1345 (1981).

${ }^{5}$ F. W. Crawford and A. B. Cannara, J. Appl. Phys. 36, 3135 (1965); W. Ott, Zeit. Naturforschung 22a, 1057 (1967).

${ }^{6}$ Y. Maron, M. D. Coleman, D. A. Hammer, and H. S. Peng, Phys. Rev. Lett. 57,699 ( $\$ 986)$.

'L. P. Block, in Physics of Auroral Arc Formation, Geophys. Monogr. Amer. Geophys. Union 25, 218 ( 1981 ).

${ }^{8}$ N. Hershkowizz, Space Sci. Rev. 41, 351 (1985), and references therein.

${ }^{2} \mathrm{~K} .3$. Armstrong and H. Fujita, in Proceedings on Second Sympasium on Plasma Double Layers, edited R. Schritweiser (Medieninhaber, Innsbruck, Austria, 1984), p. 194.

${ }^{10} \mathrm{C}$. Forest and N. Hershkowitz, J. Appl. Phys. 60, 1295 (1986).

"T. Intrator, N. Hershkowitz, and C. Chan, Phys. Fluids 27, 527 (1984).

i2. R. Smith, N. Hershkowitz, and P. Coakley, Rev. Sci. Instrum. 50, 210 (1979).

${ }^{3}$ M. H. Cho, C. Chan, N. Hershkowitz, and T. Intrator, Rev. Sci. Instrum. 55, $631(1984)$.

${ }^{14}$ E. Y. Wang, T. Intrator, and N. Hershkowitz, Rev. Sci. Instrum. 56, 519 (1985).

${ }^{15}$ F. S. Mozer, R. B. Torbert, U. V. Fahleson, C. G. Falthammar, A. Gonfalone, and A. Pedersen, IEEE Trans. Geoscience Electron. GE-16, 258 (1978); R. Grard, K. Knott, and R. Pedersen, Space Sci. Rev. 34, 289 (1983); U. W. Fahleson, M.C. Kelley, and F. S. Mozer, Planet. Space Sei. $18,1551(1970)$.

${ }^{6}$ D. Diebold, N. Hershkowitz, A. D. Bailey, N. H. Cho, T. Intrator, Rev. Sci. Instrum. 39, $270(1988)$.

${ }^{17}$ C. J. Snithells, Metals Reference Book, the ed. (Plenum, New York, $1967)$, p. 738.

${ }^{1 *} \mathrm{~N}$. Hershkowitz, Space Sci. Rev. A1, 351 (1985).

${ }^{24} \mathrm{~S}$. Brown, Basic Data of Plosma Physics, 2nd ed. (Riverside, Cambridge, MA, 1967) p. 73.

${ }^{20}$ V. L. Sizonenko, Sov. Phys. Tech. Phys. 26, 1345 (1981); G. D. Hobbs and J. A. Wesson, Plasma Phys. 9, 85 (1967). 